\title{
KATO CONSTANTS IN RIEMANNIAN GEOMETRY
}

\author{
T. BRANSON
}

\begin{abstract}
We derive improvements of Kato's inequality for sections of an irreducible $\mathrm{SO}(n)$ - or $\operatorname{Spin}(n)$-bundle $\mathbb{V}$ in the category of Riemannian manifolds, under the assumption that the section solves a first-order equivariant injectively elliptic system $\mathcal{D}$. An explicit, general (covering all $\mathbb{V}$ and $\mathcal{D}$ ) formula is given for the improved Kato constant.
\end{abstract}

\section{Introduction}

Kato's inequality for a smooth section $\varphi$ of a Riemannian vector bundle $\mathbb{V}$ with compatible connection says that

$$
|d| \varphi||^{2} \leq|\nabla \varphi|^{2} \quad \text { off } \quad\left\{x \mid \varphi_{x}=0\right\} .
$$

Here each $|\cdot|$ is computed using the Riemannian structure on the relevant bundle, and we assign the canonical tensor product metric to the bundle $T^{*} M \otimes \mathbb{V}$, where $\nabla \varphi$ lives. For some time now, a standard technique has been to use improved versions of the Kato inequality, good in the case of sections that solve some injectively elliptic system. Such an improvement should look like

$$
|d| \varphi||^{2} \leq(1-\varepsilon)|\nabla \varphi|^{2} \quad \text { off } \quad\left\{x \mid \varphi_{x}=0\right\},
$$

for some specific $\varepsilon>0$. For example, various curvatures, by virtue of Bianchitype identities, solve injectively elliptic systems, and thus satisfy improved Kato inequalities; see [10] in the case of self-dual Yang-Mills fields, and [1, 7] in the case of the Weyl curvature. These improvements are crucial to estimates at infinity of the size of these curvatures on, for example, manifolds that are asymptotically $\mathbb{R}^{n}$. J.-P. Bourguignon observed in [2] that there must be some positive Kato constant $\varepsilon$ associated to any natural injectively elliptic system. In view of applications like that in [10], and a new application to the proof of the Hijazi inequality in [5], Proposition 3.4, the determination of the best Kato constant is apparently related to the idea of Stein and Weiss [8] of determining the exponents $p$ for which $|\varphi|^{p}$ is subharmonic, $\varphi$ being a solution of the injectively elliptic system in question.

In this paper we derive an improvement of Kato's inequality for a general irreducible $\mathrm{SO}(n)$ - or $\operatorname{Spin}(n)$-bundle $\mathbb{V}$ (for $n \geq 2$ ) in the category of Riemannian manifolds, and for all equivariant (under $\mathrm{SO}(n)$ or $\operatorname{Spin}(n)$ ) first-order injectively

Received July 19, 1999. 
elliptic systems. These $\mathbb{V}$ come equipped with Riemannian metrics and compatible connections (those derived from the Levi-Civita spin connection). A change of normalization for the metric of such a $\mathbb{V}$ has no effect on the Kato constant $\varepsilon$, since only the relative normalization of the metrics on $\mathbb{V}$ and $T^{*} M \otimes \mathbb{V}$ matters, and this is fixed by the tensor product metric convention.

Let $H$ be $\mathrm{SO}(n)$ or $\operatorname{Spin}(n)$, and let $M$ be an $n$-dimensional $H$-manifold. That is, if $H=\mathrm{SO}(n)$, we require $M$ to be oriented and Riemannian, and if $H=\operatorname{Spin}(n)$, we require $M$ to be a Riemannian spin manifold. Let $\mathbb{V}=\mathcal{F} \times_{\lambda} V$ be a vector bundle canonically associated to a finite-dimensional representation $(\lambda, V)$ of $H$ and the bundle $\mathcal{F}$ of $H$-frames (i.e., oriented orthonormal frames, or Riemannian spin frames). Note that if we have spin structure, we may take $H$ to be $\operatorname{Spin}(n)$ even for $\mathrm{SO}(n)$ bundles, since we may always compose with the covering homomorphism $\operatorname{Spin}(n) \rightarrow \mathrm{SO}(n)$. Let $(\tau, T)$ be the defining representation of $\mathrm{SO}(n)$, and let $\Pi$ be an $H$-invariant projection on $T \otimes V$. $\Pi$ induces an equivariant projection, which we shall also call $\Pi$, on the bundle $T^{*} M \otimes \mathbb{V}$, since $(\tau, T)$ induces $T^{*} M \cong_{\mathrm{SO}(n)} T M$. The operator

$$
G_{\Pi}:=\Pi \circ \nabla
$$

where $\nabla$ is the $H$-equivariant covariant derivative, is then either 0 (iff $\Pi=0$ ), or an $H$-equivariant first-order differential operator from (sections of) $\mathbb{V}$ to (sections of) an $H$-equivariant direct summand $\mathbb{V}_{\Pi}$ of $T^{*} M \otimes \mathbb{V}$. It is possible to say "direct summand" because of Weyl's theorem, which insures the complete reducibility of finite-dimensional representations of $\mathfrak{s o}(n)$. If $\Theta$ is the complementary projection onto the orthogonal complement of the range of $\Pi$, then

$$
G_{\Pi}+G_{\Theta}=\nabla, \quad G_{\Pi}^{*} G_{\Pi}+G_{\Theta}^{*} G_{\Theta}=\nabla^{*} \nabla,
$$

the last equation by orthogonality of the ranges of $\Pi, \Theta$.

In fact, it is known (see $[8,6,4]$ ) that if $V$ is irreducible, then $T \otimes V$ splits into irreducibles with multiplicity one; that is,

$$
T \otimes V \cong_{H} W_{1} \oplus \cdots \oplus W_{N(V)},
$$

where

$$
W_{u} \cong_{H} W_{v} \Longleftrightarrow u=v
$$

The number $N(V)$ depends on the particular representation $(\lambda, V)$ involved; see the discussion following (13) below. Thus any projection $\Pi$ of the type discussed above has the form

$$
\Pi=\Pi_{A}:=\sum_{u \in A} \Pi_{u}
$$

for some $A \subset\{1, \ldots, N(V)\}$, where $\Pi_{u}$ is projection onto the $W_{u}$ summand. In what follows, we shall use abbreviations like

$$
G_{\Pi_{A}}=: G_{A} .
$$

Theorem 4.10 in [4] provides a complete classification, for all $(\lambda, V)$, of the sets $A$ for which $G_{A}^{*} G_{A}$ is elliptic; see Remark 6 below for a qualitative summary of 
this result. Note that

$$
\begin{aligned}
G_{\Pi}^{*} G_{\Pi} \text { elliptic } \Longleftrightarrow & \underbrace{\sigma_{1}\left(G_{\Pi}\right)(\xi)^{*} \sigma_{1}\left(G_{\Pi}\right)(\xi)} \\
& \Longleftrightarrow \sigma_{1}\left(G_{\Pi}\right)(\xi) \text { injective } \Longleftrightarrow G_{\Pi} \text { injectively elliptic, }
\end{aligned}
$$

so [4], Theorem 4.10 classifies the injectively elliptic sets $A$.

The purpose of this paper is to find improvements of the basic Kato inequality (1) on smooth sections $\varphi$ of $\mathbb{V}$. These improvements have the form (2), and are valid under the assumption that $\varphi$ is annihilated by some injectively elliptic $G_{A}$. The constant $\varepsilon=\varepsilon_{A}$ will be dependent on, and explicitly computable from, the data $(\lambda, V)$ and $A$. A formula for $\varepsilon_{A}$ is given in Theorem 4 below, and this is shown to be sharp in Theorem 7. Simpler formulas for minimal elliptic sets $A$ are special cases; these are given in various corollaries.

Let us see how these improvements come about, and in the process explain the derivation of the unimproved Kato inequality (1). Let $\varphi$ be a smooth section of $\mathbb{V}$, and let

$$
\eta:=d|\varphi|^{2}
$$

Then

$$
|\eta|^{2}=|2| \varphi|d| \varphi||^{2}=4|\varphi|^{2}|d| \varphi||^{2} \quad \text { off } \quad\left\{x \mid \varphi_{x}=0\right\} .
$$

On the other hand, we have

$$
\eta=\nabla|\varphi|^{2}=2 h^{\prime}(\varphi, \nabla \varphi),
$$

where

$$
h^{\prime}: \mathbb{V} M \times T^{*} M \otimes \mathbb{V} M \rightarrow \mathbb{V} M \otimes T^{*} M \otimes \mathbb{V} M \rightarrow T^{*} M,
$$

is the $h$-metric contraction in the two $V$ arguments. In (6), we have used the compatibility of $\nabla$; that is, the fact that our bundle metrics are parallel.

For some (not necessarily continuous) unit covector field $\xi_{0}$, we have

$$
\eta=|\eta| \xi_{0} .
$$

If $\Pi \nabla \varphi=0$, then $\eta=2 h^{\prime}(\varphi, \Theta \nabla \varphi)$, whence

$$
\begin{aligned}
|\eta| & =g^{\sharp}\left(\xi_{0}, \eta\right)=2 g^{\sharp}\left(\xi_{0}, h^{\prime}(\varphi, \Theta \nabla \varphi)\right)=2 \underline{h}\left(\xi_{0} \otimes \varphi, \Theta \nabla \varphi\right) \\
& =2 \underline{h}\left(\Theta\left(\xi_{0} \otimes \varphi\right), \nabla \varphi\right) \leq 2\left|\Theta\left(\xi_{0} \otimes \varphi\right)\right| \cdot|\nabla \varphi| .
\end{aligned}
$$

Note that $\left|\xi_{0} \otimes \varphi\right|=|\varphi|$. This shows that if there is no assumption on $\varphi$, i.e., if $\Pi=0$, then $|\eta|^{2} \leq 4|\varphi|^{2} \cdot|\nabla \varphi|^{2}$. In conjunction with (5), this gives the unimproved Kato inequality (1). The improvement comes from the fact that, for elliptic $\Pi,\left|\Theta\left(\xi_{0} \otimes \varphi\right)\right|$ is systematically smaller than $|\varphi|$. That is, a simple tensor like $\xi_{0} \otimes \varphi$ must form a certain nonzero minimum angle with the range of a co-elliptic projection like $\Theta$. An important point is that $\xi_{0}$ is real. It is irrelevant, however, whether or not there is a distinguished real form for $(\lambda, V)$. (See [8], p. 174.) 
Indeed, suppose $\varepsilon$ is an ellipticity constant for $G_{\Pi}^{*} G_{\Pi}$, in the sense that the pointwise estimate

$$
\varepsilon|\xi|^{2}|\varphi|^{2} \leq h\left(\sigma_{2}\left(G_{\Pi}^{*} G_{\Pi}\right)(\xi) \varphi, \varphi\right)
$$

holds for all covectors $\xi$ and sections $\varphi$ of $\mathbb{V}$. The leading symbol of $\nabla$ is

$$
\sigma_{1}(\nabla)(\xi) \varphi=\sqrt{-1} \xi \otimes \varphi
$$

so

$$
\varepsilon|\xi|^{2}|\varphi|^{2} \leq h\left(\sigma_{2}\left(G_{\Pi}^{*} G_{\Pi}\right)(\xi) \varphi, \varphi\right)=\left|\sigma_{1}\left(G_{\Pi}\right)(\xi) \varphi\right|^{2}=|\Pi(\xi \otimes \varphi)|^{2},
$$

whence

$$
|\varphi|^{2}=\left|\xi_{0} \otimes \varphi\right|^{2}=\underbrace{\left|\Pi\left(\xi_{0} \otimes \varphi\right)\right|^{2}}_{\geq \varepsilon|\varphi|^{2}}+\underbrace{\left|\Theta\left(\xi_{0} \otimes \varphi\right)\right|^{2}}_{\leq(1-\varepsilon)|\varphi|^{2}} .
$$

Substituting into (7), we have

$$
|\eta|^{2} \leq 4(1-\varepsilon)|\varphi|^{2}|\nabla \varphi|^{2}
$$

With (5), this gives (2).

The author learned of this problem in a lecture by Marc Herzlich, on joint work of Calderbank, Gauduchon, and Herzlich. They were able to calculate Kato constants for all minimal elliptic projections $\Pi_{A}$ in the case $N(V) \leq 4$. It was apparent that the Kato constants achieved by Calderbank, Gauduchon, and Herzlich were similar in form to the normalizing constants $\tilde{c}_{\lambda \sigma_{u}}$ of [4] (see also (15) below), which appear in our Kato constant formulas. In addition, the algebraic problems they encountered (Vandermonde systems involving conformal weights) had also appeared in the computation of the $\tilde{c}_{\lambda \sigma_{u}}$ in [4]. Thus it was natural to speculate on possible relations between the two sets of results. Shortly after the present paper was submitted, Calderbank, Gauduchon, and Herzlich were kind enough to send us a preliminary version of [5], in which the small $N(V)$ restriction is removed (though there is an exceptional class of injectively elliptic systems not covered by the main result of [5]). Remarkably, the two methods of computing the Kato constants are genuinely different; see Remark 10 below.

The author would like to thank Calderbank, Gauduchon, and Herzlich for making him aware of the Kato constant problem, and for providing a preliminary draft of [5]. He would also like to thank Peter Gilkey for supplying an elegant proof of Lemma 2, and David Calderbank for discussions clarifying the sharpness question (Remark 9 below). Discussions with Oussama Hijazi, Palle Jorgensen, and Bent Ørsted have also been helpful.

The main results of this paper, Theorems 4 and 7 , are given in the next section, as are various corollaries. In the third section, we work out a few specific examples of bundles $\mathbb{V}$ and elliptic sets $A$. 


\section{Statement and proof of the main results}

An important point is that the estimate (8) is just a statement about the representations $(\lambda, V),(\tau, T)$, their tensor product; and about the invariant inner products for these representations (given the canonical tensor product metric convention). In fact, the discussion just after (8) shows that its right side is $|\Pi(\xi \otimes \varphi)|^{2}$. Thus if $(8)$ holds at a point $x \in M$, it holds at all points; and in fact, at all points of all $n$-dimensional $H$-manifolds. We sum this up in:

\section{Lemma 1.}

(a) Suppose that there is a constant $\varepsilon$ such that for all vectors $u \in T$ and $v \in V$,

$$
\varepsilon|u|^{2}|v|^{2} \leq|\Pi(u \otimes v)|^{2} .
$$

Then on all $n$-dimensional $H$-manifolds,

$$
G_{A} \varphi=0 \quad \Rightarrow \quad|d| \varphi||^{2} \leq(1-\varepsilon)|\nabla \varphi|^{2} \text { off }\left\{x \mid \varphi_{x}=0\right\} .
$$

(b) Suppose that on some $H$-manifold $M_{0}$,

$$
\varepsilon|\xi|^{2}|\varphi|^{2} \leq|\Pi(\xi \otimes \varphi)|^{2}
$$

pointwise, for all sections $\varphi$ of $\mathbb{V}$ and all covector fields $\xi$. Then (10) holds for all $u \in T$ and $v \in V$, and as a consequence, the improved Kato inequality (11) holds on all $n$-dimensional $H$-manifolds $M$.

The second part of this will be used in combination with the following lemma to convert spectral information about the $G_{A}^{*} G_{A}$ on a special manifold (the sphere) into local information on leading symbols.

Lemma 2. On a Riemannian manifold $M$, let $D$ be a formally self-adjoint second-order differential operator on a Riemannian vector bundle $(\mathbb{V}, h)$ with leading symbol $\sigma_{2}(D)(\xi)$. Suppose we have an estimate

$$
(D \varphi, \varphi)_{L^{2}} \geq-(P \varphi, \varphi)_{L^{2}}
$$

for some formally self-adjoint differential operator $P$ of order at most one, valid on all compactly supported smooth sections $\varphi$ of $\mathbb{V}$. Then $D$ has nonnegative definite leading symbol; that is, $\sigma_{2}(D)(x, \xi)$ is a positive semidefinite element of $\operatorname{End}\left(\mathbb{V}_{x}\right)$ for any $(x, \xi)$ not in the zero section of $T^{*} M$.

Proof. Let $\varphi \in C_{\mathrm{c}}^{\infty}(\mathbb{V}), f \in C^{\infty}(M)$, and $t>0$. Applying the estimate (12) to $e^{\sqrt{-1} t f} \varphi$, dividing by $t^{2}$, and letting $t \rightarrow \infty$, we get

$$
h\left(\sigma_{2}(D)(d f) \varphi, \varphi\right)_{L^{2}} \geq 0 .
$$

Now, given $x \in M$, replace $\varphi$ by $\psi_{m} \varphi$, where $\left(\psi_{m}\right)$ is a sequence of smooth cutoff functions with $\left\|\psi_{m}\right\|_{L^{2}}=1$, but with compact supports decreasing to $\{x\}$. Taking the limit as $m \rightarrow \infty$, we get positive semidefiniteness for $\sigma_{2}(D)\left((d f)_{x}\right)$. Since each covector at $x$ is $(d f)_{x}$ for some $f$, the conclusion of the Lemma follows. 
Remark 3. If we replace $D$ by $D-\varepsilon B$, where $B$ is any second-order operator with leading symbol $|\xi|^{2} \operatorname{Id} \mathbb{V}$, the hypothesis becomes

$$
(D \varphi, \varphi)_{L^{2}} \geq \varepsilon(B \varphi, \varphi)_{L^{2}}-(P \varphi, \varphi)_{L^{2}},
$$

and the conclusion becomes

$$
\varepsilon|\xi|^{2}|\varphi|^{2} \leq h\left(\sigma_{2}(D)(x, \xi) \varphi, \varphi\right),
$$

for all sections $\varphi$ of $\mathbb{V}$ and all covector fields $\xi$.

To be specific about the modules involved in (4), we need to state the classical selection rule. First recall that weights for $\mathfrak{s o}(n)$ may be identified with elements of $\mathbb{Z}^{\ell} \cup\left(\frac{1}{2}+\mathbb{Z}\right)^{\ell}$, where $\ell=[n / 2]$. Dominant weights are those satisfying

$$
\begin{array}{ll}
\lambda_{1} \geq \lambda_{2} \geq \ldots \geq \lambda_{\ell-1} \geq \lambda_{\ell} \geq 0, & n \text { odd }, \\
\lambda_{1} \geq \lambda_{2} \geq \ldots \geq \lambda_{\ell-1} \geq\left|\lambda_{\ell}\right|, & n \text { even } .
\end{array}
$$

Isomorphism classes of finite-dimensional irreducible $\mathfrak{s o}(n)$ representations are parameterized by the set $\chi(n)$ of dominant weights in such a way that the module with parameter $\lambda$ has $\lambda$ as its (lexicographically) highest weight. We shall sometimes abuse notation, denoting a representation and its highest weight by the same letter. Thus a dominant weight $\lambda$ gives rise to a representation $(\lambda, V)$, which in turn induces a bundle which we shall call $\mathbb{V}(\lambda) . \lambda$ in $\mathbb{Z}^{\ell}$ (i.e., integral $\lambda$ ) give rise to representations of $\mathrm{SO}(n)$, and to $\mathrm{SO}(n)$ bundles; these have tensor realizations. $\lambda \in\left(\frac{1}{2}+\mathbb{Z}\right)^{\ell}$ (i.e., properly half-integral $\lambda$ ) give rise to representations of $\operatorname{Spin}(n)$ that do not factor through $\mathrm{SO}(n)$; these have tensor-spinor realizations. For more details, see for example $[8,4]$.

The selection rule is:

$$
T^{*} M \otimes \mathbb{V}(\lambda) \cong_{H} \bigoplus_{\sigma \leftrightarrow \lambda} \mathbb{V}(\sigma)
$$

where $\sigma \leftrightarrow \lambda$ if and only if $\sigma \in \chi(n)$ and

$$
\begin{aligned}
& \sigma=\lambda \pm e_{a}, \text { some } a \in\{1, \ldots, \ell\} \\
& \text { or } n \text { is odd, } \lambda_{\ell} \neq 0, \sigma=\lambda,
\end{aligned}
$$

where $e_{a}=(0, \ldots, 1,0, \ldots, 0)$ is the $a^{\text {th }}$ standard basis $\ell$-tuple. Clearly the relation $\leftrightarrow$ is symmetric. Let $\left(\sigma_{u}\right)_{u=1}^{N(\lambda)}$ be some enumeration of the $\sigma$ with $\sigma \leftrightarrow \lambda$. (A convenient way to enumerate is to put the $\sigma_{u}$ in decreasing lexicographical order. This is what we shall do in the examples of the next section, but a specific enumeration is not really needed.) As mentioned in connection with (4), the selection rule has multiplicity one: $\mathbb{V}\left(\sigma_{u}\right) \cong_{\mathfrak{s o}(n)} \mathbb{V}\left(\sigma_{v}\right) \Longleftrightarrow u=v$. Note that by (13), the number $N(\lambda)$ is bounded from above by the dimension $n$.

Let $G_{u}=G_{\lambda \sigma_{u}}$ be the gradient obtained by projecting $\nabla$ onto the $\mathbb{V}\left(\sigma_{u}\right)$ summand as in (3). To state the theorem, we shall need some further notation, and some normalizing constants from the formula for the spectrum of any $G_{\lambda \sigma_{u}}^{*} G_{\lambda \sigma_{u}}$, given in [4], Theorem 5.2. As in that reference, we always use the tensor product Riemannian structure on $T^{*} M \otimes \mathbb{V}(\lambda)$ to compute adjoints. 
Let $\rho=\rho_{\mathfrak{s o}(n)}$ be the weight given by

$$
2 \rho_{\mathfrak{s o}(n)}=(n-2, n-4, \ldots, n-2 \ell),
$$

and for any $\mathfrak{s o}(n)$ weight $\mu$, let

$$
\tilde{\mu}:=\mu+\rho .
$$

Let

$$
t(\lambda)=\left[\frac{N(\lambda)+1}{2}\right]
$$

and let

$$
s_{u}=\frac{1}{2}\left(|\tilde{\lambda}|^{2}-\left|\tilde{\sigma}_{u}\right|^{2}\right) .
$$

Note that this is easy to compute:

Let

$$
\frac{1}{2}\left(|\tilde{\lambda}|^{2}-\left|\tilde{\lambda} \pm e_{a}\right|^{2}\right)=-\frac{1}{2}\left(1 \pm 2 \tilde{\lambda}_{a}\right) .
$$

$$
\tilde{c}_{\lambda \sigma_{u}}= \begin{cases}\frac{(-1)^{t(\lambda)+1}}{\prod_{1 \leq v \leq N(\lambda), v \neq u}\left(s_{v}-s_{u}\right)}, & N(\lambda) \text { odd, } \\ \frac{(-1)^{t(\lambda)+1}}{2 \prod_{1 \leq u \leq N(\lambda),\left(\sigma_{u}\right)_{\ell}=0}\left(s_{u}+\frac{1}{2}\right)}, & n \text { even, } \lambda_{\ell}=0 \neq \lambda_{\ell-1},\left|\left(\sigma_{u}\right)_{\ell}\right|=1, \\ \frac{(-1)^{t(\lambda)}\left(s_{u}+\frac{1}{2}\right)}{\prod_{1 \leq v \leq N(\lambda), v \neq u}\left(s_{v}-s_{u}\right)}, & \text { otherwise. }\end{cases}
$$

(A unified formula handling all cases is given in [4], Remark 5.6.)

We shall also need the classical branching rule, which gives the restriction of a finite-dimensional $\mathfrak{s o}(n+1)$ representation to $\mathfrak{s o}(n)$ (imbedded in the standard way). If $\mathcal{V}(\alpha)$ is the irreducible $\mathfrak{s o}(n+1)$-module with highest weight $\alpha$, then the multiplicity of $\mathbb{V}(\lambda)$ in $\left.\mathcal{V}(\alpha)\right|_{\mathfrak{s o}(n)}$ is either 0 or 1 , and is 1 if and only if:

$$
\begin{array}{ll}
\alpha_{1} \geq \lambda_{1} \geq \cdots \geq \lambda_{\ell} \geq\left|\alpha_{\ell+1}\right|, & n \text { odd, } \\
\alpha_{1} \geq \lambda_{1} \geq \cdots \geq \alpha_{\ell} \geq\left|\lambda_{\ell}\right|, & n \text { even. }
\end{array}
$$

We shall denote this relation by $\alpha \downarrow \lambda$ or $\lambda \uparrow \alpha$. Let $L=[(n+1) / 2]$; this is the number of components in a weight of $\mathfrak{s o}(n+1)$. Let $\chi(n)$ be the set of dominant weights for $\mathfrak{s o}(n)$, and let $\alpha \in \chi(n+1), \lambda \in \chi(n), \beta \in \chi(n-1)$. $\beta$ has $L-1$ components. A remark which will be of use to us is:

$$
\begin{aligned}
& \alpha \downarrow \lambda \Rightarrow \lambda \downarrow\left(\alpha_{2}, \ldots, \alpha_{L}\right), \\
& \beta \uparrow \lambda, \alpha_{1} \in\left|\lambda_{1}\right|+\mathbb{N} \Rightarrow\left(\alpha_{1}, \beta\right) \downarrow \lambda .
\end{aligned}
$$

Here and below, $\mathbb{N}=\{0,1,2, \ldots\}$, and the $L$-tuple $\left(\alpha_{1}, \beta\right)$ is viewed as an element of $\chi(n+1)$.

A feature of the computation in [4], Sec. 5 is the distinction of certain components of a variable $\alpha \in \chi(n+1)$ with $\alpha \downarrow \lambda$. Given $\lambda$, Let $\mathcal{T}(\lambda)$ be the set of those $a$ in $\{1, \ldots, L\}$ for which $\tilde{\alpha}_{a}^{2}$ is allowed more than one value by the interlacing 
inequalities (16). It is shown in [4] that the cardinality of $\mathcal{T}(\lambda)$ is the number $t(\lambda)$ defined above in (14), which may thus be viewed as a relation between the branching complexity and selection complexity of $\lambda$. For present purposes, it is convenient to define

$$
\mathcal{T}^{\circ}(\lambda):=\{a-1 \mid a \in \mathcal{T}(\lambda)\} \backslash\{0\} .
$$

$\mathcal{T}^{\circ}(\lambda)$ is the set of component labels of a variable $\beta \in \chi(n-1)$ for which $\tilde{\beta}_{b}^{2}$ is allowed more than one value by the interlacing inequalities of the branching condition $\beta \uparrow \lambda$. With reference to (17), note that if $\beta \in \chi(n-1)$ is obtained as $\left(\alpha_{2}, \ldots, \alpha_{L}\right)$ for $\alpha \in \chi(n+1)$, then

$$
\tilde{\beta}=\left(\tilde{\alpha}_{2}, \ldots, \tilde{\alpha}_{L}\right),
$$

since $\rho_{\mathfrak{s o}(n-1)}=\left(\left(\rho_{\mathfrak{s o}(n+1)}\right)_{2}, \ldots,\left(\rho_{\mathfrak{s o}(n+1)}\right)_{L}\right)$.

Theorem 4. Suppose $G_{A}^{*} G_{A}$ is elliptic. Then

$$
\begin{aligned}
\varepsilon_{A} & =\min _{\substack{\beta \in \chi(n-1) \\
\beta \uparrow \lambda}} \sum_{u \in A} \tilde{c}_{\lambda \sigma_{u}} \prod_{b \in \mathcal{T}_{(\lambda)}^{\circ}}\left(\tilde{\beta}_{b}^{2}-s_{u}^{2}\right) \\
& =\min _{\substack{\beta \in \chi(n-1) \\
\beta \uparrow \lambda}} \sum_{u \in A}\left|\tilde{c}_{\lambda \sigma_{u}}\right| \prod_{b \in \mathcal{T}_{(\lambda)}^{\circ}}\left|\tilde{\beta}_{b}^{2}-s_{u}^{2}\right|,
\end{aligned}
$$

is an explicit, positive ellipticity constant for $G_{A}^{*} G_{A}$; that is,

$$
\varepsilon_{A}|\xi|^{2}|\varphi|^{2} \leq h\left(\sigma_{2}\left(G_{A}^{*} G_{A}\right)(x, \xi) \varphi, \varphi\right)
$$

for all sections $\varphi$ of $\mathbb{V}(\lambda)$ and all covector fields $\xi$ on any $H$-manifold. Furthermore, $\varepsilon_{A}$ is an improved Kato constant for sections annihilated by $G_{A}$ :

$$
G_{A} \varphi=0 \Rightarrow|d| \varphi||^{2} \leq\left(1-\varepsilon_{A}\right)|\nabla \varphi|^{2} \text { off }\left\{x \mid \varphi_{x}=0\right\} .
$$

Remark 5. We adopt the usual convention on empty products, so if $\mathcal{T}^{\circ}(\lambda)=\emptyset$, the product in (18) or in (19) is unity. Similarly, possible empty products in the statements of Corollaries 11-20 below are to be interpreted as unity.

Proof of the Theorem. On the standard sphere $S^{n}$, each $G_{u}^{*} G_{u}$ is diagonalized by the decomposition into $\mathfrak{s o}(n+1)$ types $\mathcal{V}(\alpha ; \lambda)$, where $\alpha$ runs over all $\mathfrak{s o}(n+1)$ dominant weights with $\alpha \downarrow \lambda$. By [3], Theorem 1.1 and [4], Theorems 4.1 and 5.2 ,

$$
\begin{aligned}
\operatorname{eig}\left(\nabla^{*} \nabla, \mathcal{V}(\alpha ; \lambda)\right) & =|\tilde{\alpha}|^{2}-\left|\rho_{\mathfrak{s o}(n+1)}\right|^{2}-|\tilde{\lambda}|^{2}+\left|\rho_{\mathfrak{s o}(n)}\right|^{2} \\
\operatorname{eig}\left(G_{u}^{*} G_{u}, \mathcal{V}(\alpha ; \lambda)\right) & =\tilde{c}_{\lambda \sigma_{u}} \prod_{a \in \mathcal{T}(\lambda)}\left(\tilde{\alpha}_{a}^{2}-s_{u}^{2}\right)
\end{aligned}
$$

Note that among all the $\tilde{\alpha}_{a}^{2}$, only $\tilde{\alpha}_{1}^{2}$ is allowed infinitely many values by the branching rule (16); these values,

$$
\left(\lambda_{1}+\frac{n-1}{2}+j\right)^{2}, \quad j \in \mathbb{N},
$$


form an increasing sequence tending to infinity. By (21),

$$
\begin{aligned}
\operatorname{eig}\left(\nabla^{*} \nabla, \mathcal{V}(\alpha ; \lambda)\right) & \leq \tilde{\alpha}_{1}^{2}+\text { const } \\
\operatorname{eig}\left(G_{u}^{*} G_{u}, \mathcal{V}(\alpha ; \lambda)\right) & \geq C(\lambda, u, \alpha) \tilde{\alpha}_{1}^{2}-\mathrm{const}
\end{aligned}
$$

where

$$
C(\lambda, u, \alpha):=\tilde{c}_{\lambda \sigma_{u}} \prod_{\substack{a \in \mathcal{T}(\lambda) \\ a \neq 1}}\left(\tilde{\alpha}_{a}^{2}-s_{u}^{2}\right) .
$$

In (22) and below, the value of "const" changes from line to line, and depends on $\lambda$. The coefficients $C(\lambda, u, \alpha)$ must be nonnegative, to avoid contradicting the positive semidefiniteness of $G_{u}^{*} G_{u}$. They are also bounded above for fixed $\lambda$, since $\left(\tilde{\alpha}_{2}, \ldots, \tilde{\alpha}_{L}\right)$ and $u$ run through finite sets. Thus

$$
\begin{aligned}
\operatorname{eig}\left(G_{u}^{*} G_{u}, \mathcal{V}(\alpha ; \lambda)\right) & \geq C(\lambda, u, \alpha) \operatorname{eig}\left(\nabla^{*} \nabla, \mathcal{V}(\alpha ; \lambda)\right)-\text { const }, \\
\operatorname{eig}\left(G_{A}^{*} G_{A}, \mathcal{V}(\alpha ; \lambda)\right) & \geq\left(\sum_{u \in A} C(\lambda, u, \alpha)\right) \operatorname{eig}\left(\nabla^{*} \nabla, \mathcal{V}(\alpha ; \lambda)\right)-\mathrm{const},
\end{aligned}
$$

and we have an estimate of the form (12), with

$$
D=G_{A}^{*} G_{A}-\varepsilon \nabla^{*} \nabla, \quad \varepsilon=\min _{\alpha \downarrow \lambda} \sum_{u \in A} C(\lambda, u, \alpha), \quad P=\text { const. }
$$

To convert this constant to the form (18), note that for $\alpha \in \chi(n+1)$ and $\lambda \in \chi(n)$, we have

$$
\beta=\left(\beta_{1}, \ldots, \beta_{L-1}\right):=\left(\alpha_{2}, \ldots, \alpha_{L}\right) \in \chi(n-1),
$$

and by (17),

$$
\alpha \downarrow \lambda \quad \Longleftrightarrow \quad \beta \uparrow \lambda \text { and } \alpha \in \lambda_{1}+\mathbb{N} \text {. }
$$

The improved Kato inequality, with constant given by (18), now follows from Lemmas 1 and 2, and the aforementioned nonnegativity of $C(\lambda, u, \alpha)$ gets us from (18) to (19).

It remains to show the positivity of $\varepsilon_{A}$. We claim that $\sum_{u \in A} C(\lambda, u, \alpha)>0$ for each $\alpha$. Assuming the contrary for some $\alpha$, we get an infinite-dimensional null space for $G_{A}^{*} G_{A}$, since this null space must contain all $\mathcal{V}(\gamma ; \lambda)$ with $\left(\gamma_{2}, \ldots, \gamma_{L}\right)=$ $\left(a_{2}, \ldots, \alpha_{L}\right)$. This contradicts the ellipticity of $G_{A}^{*} G_{A}$. As a result, $\varepsilon_{A}>0$ is assured.

Remark 6. By [4], Theorem 4.10, given $\lambda$, a set $A \subset\{1, \ldots, N(\lambda)\}$ is elliptic if and only if it contains at least one of a certain explicitly described list of subsets $B_{i}(\lambda)$ of $\{1, \ldots, N(\lambda)\}$. We shall call the $B_{i}(\lambda)$ minimal elliptic sets. Each $B_{i}(\lambda)$ has cardinality 1 or 2 , and depending on $\lambda$ either (i) the $B_{i}(\lambda)$ partition $\{1, \ldots, N(\lambda)\}$, (ii) the $B_{i}(\lambda)$ partition $\{1, \ldots, N(\lambda)\} \backslash\{u\}$ for some $u$, or (iii) the union of the $B_{i}(\lambda)$ is $\{1, \ldots, N(\lambda)\}$, and the $B_{i}(\lambda)$ are disjoint except for one pair of doubleton sets which intersects in one element. 
Theorem 7. If $A$ is elliptic, then the $\varepsilon_{A}$ of Theorem 4 is the largest ellipticity constant for $G_{A}^{*} G_{A}$ at any point of any $H$-manifold. $\varepsilon_{A}$ is also the largest universal Kato constant for $G_{A}$.

Proof. Let $\beta$ be an $\mathfrak{s o}(n-1)$-type realizing the minimum in (18). If there is a better ellipticity constant $\varepsilon>\varepsilon_{A}$, then

$$
G_{A}^{*} G_{A}-\frac{1}{2}\left(\varepsilon+\varepsilon_{A}\right) \nabla^{*} \nabla
$$

has positive definite leading symbol, but, when realized on standard $S^{n}$, is negative definite on the infinite-dimensional space

$$
\bigoplus_{j=0}^{\infty} \mathcal{V}\left(\left(\left|\lambda_{1}\right|+j, \beta\right) ; \lambda\right)
$$

(The absolute value bars on $\lambda_{1}$ in (23) are needed only to cover the case $n=2$.) This contradiction shows that $\varepsilon_{A}$ is sharp in (20) on the sphere. Since ellipticity constants for operators of the form $G_{A}^{*} G_{A}$ are universal in the sense of the discussion before Lemma $1, \varepsilon_{A}$ is a sharp ellipticity constant at each point $x$ of any $H$-manifold $M$.

The above results on ellipticity constants may be rephrased as follows. Given a real unit vector $v$ in the defining representation $(\tau, T)$ of $\mathfrak{s o}(n)$, let $\zeta(v)$ be left tensor product by $v$. Then the norm of the self-adjoint linear transformation $\zeta(v)^{*} \Theta \zeta(v)$ on the space $V$ of the representation $(\lambda, V)$ is $1-\varepsilon_{A}$. In fact, the branching decomposition relative to the subgroup $H_{v}$ of $H$ fixing $v$ diagonalizes $\zeta(v)^{*} \Theta \zeta(v)$, and so the norm (or highest eigenvalue) is attained on a punctured $H_{v}$-submodule $V_{v}$ of $V$. The list of eigenvalues, with multiplicities, is independent of $v$.

If $\Theta=0$, then $\varepsilon_{A}=1$ is clearly a sharp Kato constant. Otherwise, pick a unit vector $\alpha$ in $V_{v}$, and let $\beta:=\Theta(v \otimes \alpha)=\Theta \zeta(v) \alpha$. Since $\Theta \neq 0$, we have $\beta \neq 0$. We use $\alpha$ and $\beta$ to define corresponding parallel sections over flat $\mathbb{R}^{n}$, which we shall also denote by $\alpha, \beta$. If $\beta=\sum_{i=1}^{n} d x^{i} \otimes \beta_{i}$, let

$$
\varphi:=\alpha+\sum_{i=1}^{n} \beta_{i} x^{i} .
$$

Since $\nabla \varphi=\beta$ and $\beta$ lies in the range of $\Theta$, we have $G_{A} \varphi=0$. In the notation of Section 1, at the origin, $\eta=2 \operatorname{Re} h^{\prime}(\alpha, \beta)$. Of all real unit vectors $\xi$ in $T$, the choice $\xi=\xi_{0}$ is the unique one which maximizes $g^{\sharp}(\eta, \xi)$. But

$$
\begin{aligned}
g^{\sharp}(\eta, \xi) & =2 \operatorname{Re} \underline{h}(\xi \otimes \alpha, \beta) \\
& =2 \operatorname{Re} \underline{h}(\xi \otimes \alpha, \Theta(v \otimes \alpha)) \\
& =2 \operatorname{Re} \underline{h}(\Theta(\xi \otimes \alpha), \Theta(v \otimes \alpha)) \\
& \leq 2|\Theta(\xi \otimes \alpha)||\Theta(v \otimes \alpha)| .
\end{aligned}
$$


By the choice of $\alpha$ and the fact that the eigenvalue list for $\zeta(v)^{*} \Theta \zeta(v)$ is independent of $v$, we have $\left|\Theta\left(\xi_{0} \otimes \alpha\right)\right| \leq|\Theta(v \otimes \alpha)|$. Thus

$$
2|\Theta(v \otimes \alpha)|^{2}=g^{\sharp}(\eta, v) \leq g^{\sharp}\left(\eta, \xi_{0}\right) \leq 2\left|\Theta\left(\xi_{0} \otimes \alpha\right)\right||\Theta(v \otimes \alpha)| \leq 2|\Theta(v \otimes \alpha)|^{2},
$$

forcing

$$
\left|\Theta\left(\xi_{0} \otimes \alpha\right)\right|=|\Theta(v \otimes \alpha)| .
$$

If $\xi_{0} \neq v$, take $a_{0} \in H$ with $\tau\left(a_{0}\right) \xi_{0}=v$. By (24) and the $H$-invariance of all the metrics and of $\Theta$,

$$
\left|\Theta\left(v \otimes \lambda\left(a_{0}\right) \alpha\right)\right|=\left|\Theta\left((\tau \otimes \lambda)\left(a_{0}\right)\left(\xi_{0} \otimes \alpha\right)\right)\right|=\left|\Theta\left(\xi_{0} \otimes \alpha\right)\right|=|\Theta(v \otimes \alpha)| .
$$

Since $V_{v}$ is invariant under the subgroup $H_{v}$ of $H$ fixing $v$, equation (25) holds for the group generated by $a_{0}$ and $H_{v}$, and thus for all $a \in H$. This gives $\operatorname{span}(\lambda(H) \alpha) \subset V_{v}$, so $V_{v}=V$, by the $H$-irreducibility of $(\lambda, V)$. In this event, $\zeta(v)^{*} \Pi \zeta(v)$ and $\zeta(v)^{*} \Theta \zeta(v)$ are positive multiples of the identity, so the bilinear form $\operatorname{Re} \underline{h}(\Theta(\xi \otimes \alpha), \Theta(v \otimes \alpha))$ is a multiple of $g^{\sharp}(\xi, v)$. But then $\xi=v$ maximizes the functional $g^{\sharp}(\xi, v)$, forcing $\xi_{0}=v$ after all.

Having established that $\xi_{0}=v$, we have equality in the inequalities of (9), and in the Schwarz inequality step of (7). This shows that an improved Kato inequality with a constant larger than $\varepsilon_{A}$ fails in $\mathbb{R}^{n}$.

Remark 8. The case where $\zeta(v)^{*} \Pi \zeta(v)$ and $\zeta(v)^{*} \Theta \zeta(v)$ are positive multiples of the identity can actually occur. For example, let $(\lambda, V)$ be the spin representation in odd dimension, or one of the half-spin representation in even dimensions, and let $\Pi$ be projection onto the spinor (resp. twistor) summand of $T \otimes V$; then $\Theta$ is projection onto the twistor (resp. spinor) summand.

Remark 9. For a general metric $g$, there need not be any solutions of an injectively elliptic system $G_{A} \varphi=0$, even locally. Thus the statement in Theorem 7 about universal Kato constants is the appropriate one. The only other sensible sharpness question for Kato constants concerns solutions at a point $x$, which always exist because $(\nabla \varphi)_{x}$ is arbitrarily prescribable. If $A$ is elliptic, $\left(G_{A} \varphi\right)_{x}=0$, and $\varphi_{x} \neq 0$, then $\left|(d|\varphi|)_{x}\right|^{2} \leq\left(1-\varepsilon_{A}\right)\left|(\nabla \varphi)_{x}\right|^{2}$. In this statement, $\varepsilon_{A}$ is sharp for any metric and at any point, by a version of the $\mathbb{R}^{n}$ argument above, using normal coordinates.

Remark 10. The approach of [5] to the Kato constants is essentially different from ours. Their formula is an extremum over the selection rule (13) rather than (as in our formula) over the branching rule. More precisely, they extremize over maximal non-elliptic sets $A$. (Theorem 4.10 of [4], by identifying the minimal elliptic sets, also implicitly identifies the maximal non-elliptic sets.) Their approach requires fairly explicit knowledge of the action of the central character of $\mathfrak{s o}(n)$ in each representation - that is, knowledge of the values of the higher Casimir elements in the enveloping algebra. The corresponding power in our argument derives from the spectral calculations in [4], which encode central character information (as well as additional information) more implicitly. In 
fact, one induces all the way up to $\mathfrak{s o}(n+1,1)$ to gather the information, so that four "levels," $\mathfrak{s o}(n-1), \mathfrak{s o}(n), \mathfrak{s o}(n+1)$, and $\mathfrak{s o}(n+1,1)$, get involved in the combination of [4] and the present arguments.

There is an exceptional case in which [5] is not able to identify a sharp Kato constant: $n$ and $N$ odd, and $\lambda$ properly half-integral. The technical difficulty is a paucity of non-elliptic sets over which to take the extremum. To speculate somewhat, this may be a symptom of a general fact about induced representations: the branching rule at a given $\lambda$ is somewhat more powerful than the selection rule at $\lambda$, since it encodes information about all invariant differential operators, rather than just low-order ones. The cure for this, within the [5] setting, may be to bring in information on the selection rule at $\lambda$-selection targets $\sigma$; that is, to iterate the selection rule in order to make contact with information on higher-order operators.

In the following corollaries, we shall be interested in simplified statements of special cases of Theorem 4. Each minimal elliptic set, for each $\lambda$, is covered by one or another of the corollaries.

Corollary 11. For sections annihilated by the top gradient $G_{1}$ valued in $\mathbb{V}(\lambda+$ $\left.e_{1}\right)$, we have an improved Kato inequality with constant

$$
\varepsilon_{1}=\tilde{c}_{\lambda, \lambda+e_{1}} \prod_{b \in \mathcal{T}^{\circ}(\lambda)}\left(\left(\tilde{\lambda}_{b}-\frac{1}{2}\right)^{2}-\left(\tilde{\lambda}_{1}+\frac{1}{2}\right)^{2}\right) .
$$

Proof. By (19), the minimum is attained when each $\left|\tilde{\beta}_{b}\right|$ is as close to $\left|s_{1}\right|$ as possible. By the branching rule,

$$
\left|\tilde{\beta}_{b}\right| \leq\left|\tilde{\lambda}_{b}-\frac{1}{2}\right|<\left|\tilde{\lambda}_{1}+\frac{1}{2}\right|=\left|s_{1}\right|,
$$

with equality in the $\leq$ step iff $\left|\beta_{b}\right|=\left|\lambda_{b}\right|$. This evaluates the minimum in Theorem 4, and gives (26).

Remark 12. The quantities $\tilde{\lambda}_{b}-\frac{1}{2}$ and $\tilde{\lambda}_{1}+\frac{1}{2}$ appearing in (26) are (plus or minus) the $s_{u}$ of various gradient targets. After substituting the appropriate $s_{u}$, one may cancel various terms with terms appearing in $\tilde{c}_{\lambda \sigma_{1}}$ to re-express $\varepsilon_{1}$ more simply in terms of sums and differences of various pairs of $s_{u}$. Similar considerations hold for the expressions in the corollaries below.

There are three exceptional (non-top) classes of singleton $G_{u}^{*} G_{u}$ which are elliptic. The first is described in [4], Theorem 4.10(b); the corresponding Kato constant result is:

Corollary 13. Suppose $n$ is even, $\left|\lambda_{\ell}\right| \geq 1$, and $\left|\left(\sigma_{u}\right)_{\ell}\right|=\left|\lambda_{\ell}\right|-1$. Sections annihilated by $G_{u}$ satisfy an improved Kato inequality with constant

$$
\varepsilon_{u}=\tilde{c}_{\lambda \sigma_{u}} \prod_{b \in \mathcal{T}(\lambda)^{\circ}}\left(\left(\tilde{\lambda}_{b+1}+\frac{1}{2}\right)^{2}-\left(\left|\lambda_{\ell}\right|-\frac{1}{2}\right)^{2}\right) .
$$


Proof. Here

$$
s_{u}=\left|\lambda_{\ell}\right|-\frac{1}{2},
$$

and we make the factors $\left|\beta_{b}^{2}-s_{u_{0}}^{2}\right|$ from (19) minimal by choosing $\beta_{b}=\left|\lambda_{b+1}\right|$ for all $b \in \mathcal{T}(\lambda)^{\circ}$.

The second and third exceptional classes are described in [4], Theorem 4.10(c). The Kato constant results are given in the next two corollaries.

Corollary 14. Suppose

$$
\begin{gathered}
n \text { is odd, } \lambda_{\ell}=\frac{1}{2}, \sigma_{u}=\lambda, \\
\text { or } \quad n \text { is even, }\left|\lambda_{\ell}\right|=\frac{1}{2},\left(\sigma_{u}\right)_{\ell}=-\lambda_{\ell} .
\end{gathered}
$$

Sections annihilated by $G_{u}$ satisfy an improved Kato inequality with constant

$$
\varepsilon_{u}=\tilde{c}_{\lambda \sigma_{u}} \prod_{b \in \mathcal{T}(\lambda)^{\circ}}\left(\tilde{\lambda}_{b+1}+\frac{1}{2}\right)^{2} .
$$

The proof is similar to that of Corollary 13; note that here, $s_{u}=0$.

Corollary 15. Suppose $n$ is odd, $\lambda_{\ell} \geq \frac{3}{2}$ is properly half-integral, and $\sigma_{u}=\lambda$. Sections annihilated by $G_{u}$ satisfy an improved Kato inequality with constant

$$
\varepsilon_{u}=\tilde{c}_{\lambda \lambda} \prod_{b \in \mathcal{T}(\lambda)^{\circ}}\left(\tilde{\lambda}_{b+1}+\frac{1}{2}\right)^{2}
$$

where we set $\tilde{\lambda}_{\ell+1}=0$ by convention.

The proof is again similar; we just need to note that $\ell$ is now in $\mathcal{T}(\lambda)^{\circ}$, and the optimal value of $\tilde{\beta}_{\ell}$ is $1 / 2$.

Remark 16. If the gradient targets are numbered in decreasing lexicographical order, then the $\sigma_{u}$ described in Corollaries 13 and 14 is is $\sigma_{t_{\lambda}+1}$, while the one described in Corollary 15 is $\sigma_{t_{\lambda}}$.

The typical case of a minimal elliptic set of cardinality 2 is described in the following corollary.

Corollary 17. Suppose

$$
\lambda_{a}>\left|\lambda_{a+1}\right|, \quad a<\ell .
$$

Then $A=\{u, v\}$, where $\sigma_{u}=\lambda-e_{a}$ and $\left|\left(\sigma_{v}\right)_{a+1}\right|=\left|\lambda_{a+1}\right|+1$, is a minimal elliptic set, and sections annihilated by $G_{A}$ satisfy an improved Kato inequality with constant

$$
\varepsilon_{A}=\left(\left(\tilde{\lambda}_{a}-\frac{1}{2}\right)^{2}-\left(\left|\tilde{\lambda}_{a+1}\right|+\frac{1}{2}\right)^{2}\right) \min \{-X, Y\}
$$


where

$$
\begin{gathered}
X=\tilde{c}_{\lambda \sigma_{u}}\left(\prod_{\substack{b \in \mathcal{T}^{\circ}(\lambda) \\
b<a}}\left(\left(\tilde{\lambda}_{b+1}+\frac{1}{2}\right)^{2}-\left(\tilde{\lambda}_{a}-\frac{1}{2}\right)^{2}\right)\right) \\
\prod_{\substack{b \in \mathcal{T}^{\circ}(\lambda) \\
b>a}}\left(\left(\tilde{\lambda}_{b}-\frac{1}{2}\right)^{2}-\left(\tilde{\lambda}_{a}-\frac{1}{2}\right)^{2}\right), \\
Y=\tilde{c}_{\lambda \sigma_{v}}\left(\prod_{\substack{b \in \mathcal{T}^{\circ}(\lambda) \\
b<a}}\left(\left(\tilde{\lambda}_{b+1}+\frac{1}{2}\right)^{2}-\left(\left|\tilde{\lambda}_{a+1}\right|+\frac{1}{2}\right)^{2}\right)\right) \\
\prod_{\substack{b \in \mathcal{T}^{\circ}(\lambda) \\
b>a}}\left(\left(\tilde{\lambda}_{b}-\frac{1}{2}\right)^{2}-\left(\left|\tilde{\lambda}_{a+1}\right|+\frac{1}{2}\right)^{2}\right) .
\end{gathered}
$$

Remark 18. The rather strange way in which $\sigma_{v}$ is identified is necessary in order to cover the case $n$ even, $a+1=\ell, \lambda_{\ell} \leq 0$. Note that in this case, if $\lambda_{\ell}=0$, we get two choices for $\left(\sigma_{v}\right)_{\ell}$, namely \pm 1 .

Proof of Corollary 17. A is a minimal elliptic set by [4], Theorem 4.10, Cases d and e. To have a chance of attaining the minimum in (19), we must choose

$$
\beta_{b}= \begin{cases}\lambda_{b+1}, & b<a \\ \lambda_{b}, & b>a,\end{cases}
$$

since

$$
s_{u}=\tilde{\lambda}_{a}-\frac{1}{2}, \quad s_{v}=-\left(\left|\tilde{\lambda}_{a+1}\right|+\frac{1}{2}\right) .
$$

Only the value of $\beta_{a}$ is not immediately clear. However, given (29), the quantity to be minimized in (18) is now a quadratic function of the single variable $\tilde{\beta}_{a}$, as this variable ranges over the set

$$
\left\{\left|\lambda_{a+1}\right|+\frac{1}{2}(n-1-2 a), \ldots, \lambda_{a}+\frac{1}{2}(n-1-2 a)\right\} .
$$

This quadratic function $Q\left(\tilde{\beta}_{a}\right)$ has its extremum (over all real values of $\tilde{\beta}_{a}$ ) at 0 . Thus, depending on the sign of the lead coefficient of $Q$, the minimum is achieved at one of the extreme values of the set (30). When we substitute either of these values into the two-term sum in (18), one of the terms vanishes. The result is (27).

Remark 19. The optimal choice of $\tilde{\beta}_{a}$ in the proof of Corollary 17 is

$$
\tilde{\beta}_{a}= \begin{cases}\left|\lambda_{a+1}\right| & \text { if } Y \geq-X \\ \lambda_{a} & \text { if } Y \leq-X\end{cases}
$$

(By the nonnegativity of the $C(\lambda, u, \alpha)$ and (28), $-X$ and $Y$ are both positive.)

There is another sort of minimal elliptic set of cardinality 2; this is Case $\mathbf{f}$ of [4], Theorem 4.10. Its Kato constant is described by the following corollary. 
Corollary 20. Suppose $n$ is odd and $\lambda_{\ell} \in \mathbb{Z}^{+}$. Then $A=\{u, v\}$ with $\sigma_{u}=\lambda$, $\sigma:=\sigma_{v}=\lambda-e_{\ell}$ is a minimal elliptic set, and

$$
G_{A} \varphi=0 \Rightarrow|d| \varphi||^{2} \leq\left(1-\varepsilon_{A}\right)|\nabla \varphi|^{2} \text { off }\left\{x \mid \varphi_{x}=0\right\}
$$

with

$$
\varepsilon_{A}=\lambda_{\ell}^{2} \min \{-X, Y\}
$$

where

$$
\begin{aligned}
& X=\tilde{c}_{\lambda \sigma} \prod_{\substack{b \in \mathcal{T}^{\circ}(\lambda) \\
b<\ell}}\left(\left(\tilde{\lambda}_{b+1}+\frac{1}{2}\right)^{2}-\lambda_{\ell}^{2}\right), \\
& Y=\tilde{c}_{\lambda \lambda} \prod_{\substack{b \in \mathcal{T}^{\circ}(\lambda) \\
b<\ell}}\left(\tilde{\lambda}_{b+1}+\frac{1}{2}\right)^{2} .
\end{aligned}
$$

Proof. $A$ is a minimal elliptic set by Case $\mathbf{f}$ of [4], Theorem 4.10. Since $s_{u}=0$ and

$$
s_{v}=\tilde{\lambda}_{\ell}-\frac{1}{2}=\lambda_{\ell}
$$

we only have a chance of achieving the minimum in (19) if we choose $\beta_{b}=\lambda_{b+1}$ for all $b \in \mathcal{T}^{\circ}(\lambda)$ with $b<\ell$. Given this choice, (18) is now a quadratic $Q\left(\tilde{\beta}_{\ell}\right)$ in the single variable $\tilde{\beta}_{\ell}$. As in the proof of Corollary 17 , we must achieve the minimum at $\tilde{\beta}_{b}=0$ or $\tilde{\beta}_{b}=\lambda_{\ell}$. Substituting either of these values causes one of the two terms of the sum in (18) to vanish; the result is (31).

\section{Examples}

We shall typically order the $\sigma_{u}$ in decreasing lexicographical order, so that $\sigma_{1}$ is always the "top target" $\lambda+e_{1}$. In the notation $\varepsilon_{A}$, if $A$ is a singleton $\{u\}$, we shall write $\varepsilon_{u}$ instead of $\varepsilon_{\{u\}}$. We shall also abbreviate $\tilde{c}_{\lambda \sigma_{u}}$ as $\tilde{c}_{u}$.

In [5], Calderbank, Gauduchon, and Herzlich record the values of the sharp Kato constants for all minimal elliptic sets, in all cases where $N(\lambda) \leq 4$. (This includes the small $N(\lambda)$ cases which are not covered by their main argument; see Remark 10 above.) In the originally submitted version of the present paper, all $N(\lambda) \leq 4$ cases were treated in detail as examples; that is, the above corollaries were used to write the sharp Kato constants in terms of the entries of $\lambda$. Since this information is now available in [5], we omit it here in the interest of brevity; however, the calculations are available to interested readers upon request.

One case of special interest is that of algebraic Weyl tensors for $n \geq 4$; these are totally trace-free 4 -tensors $Y$ with the symmetries

$$
Y_{i j k l}=Y_{k l i j}=-Y_{j i k l}=-Y_{i k l j}-Y_{i l j k} .
$$

If $n \geq 5$, then the algebraic Weyl tensors comprise a bundle isomorphic to $\mathbb{V}(2,2,0, \ldots, 0)$, while for $n=4$, they are $\mathbb{V}(2,2) \oplus \mathbb{V}(2,-2)$; the direct summands are the self- and anti-self-dual algebraic Weyl tensors. (See [9] for details.) 
If $n \geq 7$, the algebraic Weyl tensors have $N(\lambda)=3$, the targets being $\sigma_{1}=$ $\lambda+e_{1}, \sigma_{2}=\lambda+e_{3}$, and $\sigma_{3}=\lambda-e_{2}$. The minimal elliptic sets are $\{1\}$ and $\{2,3\}$ by [4], Theorem $4 \cdot 10(\mathbf{a}, \mathbf{d})$. By Corollary 17 ,

$$
\varepsilon_{\{2,3\}}=\frac{2}{n+1} .
$$

This is especially interesting because the Weyl tensor of the metric is automatically annihilated by $G_{2}$, by virtue of the second Bianchi identity [9]. Since $G_{3}$ is realized, up to a constant multiple, by the divergence $\nabla^{i} Y_{i j k l}$, we get the result that for $n \geq 7$, if the metric Weyl tensor is divergence-free, then it satisfies a Kato inequality with $\varepsilon=2 /(n+1)$. The divergence-free condition can in turn be enforced by the vanishing of the $\mathbb{V}(2,1,0, \ldots, 0)$ part of $\nabla b$, where $b$ is the trace-free Ricci tensor, by the contracted second Bianchi identity: if $\rho$ is the Ricci tensor, $\tau$ the scalar curvature, and

$$
\mathrm{P}:=\frac{1}{n-2}\left(\rho-\frac{1}{2(n-1)} \tau g\right)
$$

then

$$
\nabla^{i} C_{i j k l}=(n-3)\left(\nabla_{k} \mathrm{P}_{j l}-\nabla_{l} \mathrm{P}_{j k}\right) .
$$

(The scalar curvature part of $\mathrm{P}$ influences the right side of (32), but not the $\mathbb{V}(2,1,0, \ldots, 0)$ part of this right side.) As a special case of this, the Weyl tensor on an Einstein manifold satisfies a Kato inequality with $\varepsilon=2 /(n+1)$.

If $n=6$, the algebraic Weyl tensors have $N(\lambda)=4$, with gradient targets $\sigma_{1}=\lambda+e_{1}, \sigma_{2}=\lambda+e_{3}, \sigma_{3}=\lambda-e_{3}, \sigma_{4}=\lambda-e_{2}$. By [4], Theorem 4.10(a,d,e), the minimal elliptic sets are $\{1\},\{2,4\}$, and $\{3,4\}$. The analogue of the above $(n \geq 7)$ discussion involves the non-minimal elliptic set $\{2,3,4\}$, since the second Bianchi identity guarantees the absence of the $\mathbb{V}(2,2,1) \oplus \mathbb{V}(2,2,-1)$ part of $\nabla C$ when $C$ is the Weyl tensor of the metric. Appending the divergence-free condition (absence of the $\mathbb{V}(2,1,0)$ part), and returning to Theorem 4 to chase through the non-minimal calculation, we get $\varepsilon_{\{2,3,4\}}=2 / 7$, continuing the above formula $\varepsilon=2 /(n+1)$ from higher dimensions. (If we require only that the $\mathbb{V}(2,2,1)$ or $\mathbb{V}(2,2,-1)$ part of a divergence-free algebraic Weyl tensor vanish, we get the slightly worse Kato constant $\varepsilon_{\{2,4\}}=\varepsilon_{\{3,4\}}=1 / 4$.)

If $n=5$, the algebraic Weyl tensors have $N(\lambda)=3$, with gradient targets $\lambda+e_{1}, \lambda$, and $\lambda-e_{2}$. The minimal elliptic sets are $\{1\}$ and $\{2,3\}$ by [4], Theorem $4.10(\mathbf{a}, \mathbf{f})$. The second Bianchi identity guarantees the vanishing of the $\mathbb{V}(2,2)$ part of $\nabla C$, for $C$ the metric Weyl tensor, and the imposition of the divergence free condition implies a Kato inequality on $C$ with constant $\varepsilon_{\{2,3\}}=1 / 3$, again continuing the formula $2 /(n+1)$ from higher dimensions.

Let $n=4$ and $\lambda=(2, \pm 2)$. Here $N(\lambda)=2$ with targets $\sigma_{1}=(3, \pm 2)$ and $\sigma_{2}=(2, \pm 1)$. By [4], Theorem $4.10(\mathbf{a}, \mathbf{b}),\{1\}$ and $\{2\}$ are elliptic sets. By Corollary 13,

$$
\varepsilon_{2}=\tilde{c}_{2}=\frac{2}{5},
$$


again continuing the formula $2 /(n+1)$ from higher dimensions. The value of $\varepsilon_{2}$ figures in work of Gursky and LeBrun [7]. Note that a condition guaranteeing that either the self- or anti-self-dual part of the Weyl tensor $\left(C_{+}\right.$or $\left.C_{-}\right)$is divergence free will enforce an improved Kato inequality on that part. For example, if the part of $\nabla_{k} b_{j l}-\nabla_{l} b_{j k}$ which is self-dual in its two-form $(k l)$ argument vanishes, then $C_{+}$satisfies the improved Kato inequality.

Another interesting example is the Rarita-Schwinger operator. This is the self-gradient on the twistors $\mathbb{V}\left(\frac{3}{2}, \frac{1}{2}, \ldots, \frac{1}{2}\right)$ when $n$ is odd, and the gradients interchanging the bundles $\mathbb{V}\left(\frac{3}{2}, \frac{1}{2}, \ldots, \frac{1}{2}, \pm \frac{1}{2}\right)$ when $n \geq 4$ is even. Suppose for the moment that $n \neq 3$. For any of these irreducible twistor bundles, we have $N(\lambda)=4$. A Rarita-Schwinger operator is elliptic by [4], Theorem 4.10(c), and is $G_{3}$ in our numerology. Twistors annihilated by a Rarita-Schwinger operator satisfy a Kato inequality with constant

$$
\varepsilon_{3}=\frac{n-2}{n(n+2)}
$$

by Corollary 14 . When $n=3$, the Kato constant for the Rarita-Schwinger operator is $\varepsilon_{2}=1 / 15$ by Corollary 15 ; this continues the formula $(n-2) / n(n+2)$ from higher dimensions.

\section{References}

[1] S. Bando, A. Kasue, and H. Nakajima, On a construction of coordinates at infinity on manifolds with fast curvature decay and maximal volume growth, Invent. Math. 97 (1989), 313-349.

[2] J.-P. Bourguignon, The magic of Weitzenböck formulas, in Variational Methods (Paris 1988), H. Berestycki, J.-M. Coron, I. Ekeland, eds., PNLDE, vol. 4, Birkhäuser Boston, Boston, MA, 1990, pp. 251-271.

[3] T. Branson, Harmonic analysis in vector bundles associated to the rotation and spin groups, J. Funct. Anal. 106 (1992), 314-328.

[4] _ Stein-Weiss operators and ellipticity, J. Funct. Anal. 151 (1997), 334-383.

[5] D. Calderbank, P. Gauduchon, and M. Herzlich, Refined Kato inequalities and conformal weights in Riemannian geometry. Preprint, xxx archive, math.DG/9909116, to appear in J. Funct. Anal.

[6] H. Fegan, Conformally invariant first order differential operators, Quart. J. Math. Oxford Ser. 27 (1976), 371-378.

[7] M. Gursky and C. LeBrun, On Einstein manifolds of positive sectional curvature, Ann. Global Anal. Geom. 17 (1999), 315-328.

[8] E. Stein and G. Weiss, Generalization of the Cauchy-Riemann equations and representations of the rotation group, Amer. J. Math. 90 (1968), 163-196.

[9] R. Strichartz, Linear algebra of curvature tensors and their covariant derivatives, Canad. J. Math. 40 (1988), 1105-1143.

[10] K. Uhlenbeck, Removable singularities for Yang-Mills fields, Comm. Math. Phys. 83 (1982), 11-30.

Department of Mathematics, The University of Iowa, Iowa City IA 52242

E-mail address: branson@math.uiowa.edu 